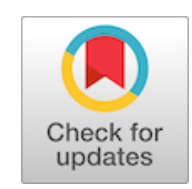

\title{
Impacto de la participación del residente y su rol en desenlaces clínicos de los pacientes llevados a colecistectomía en un hospital universitario
}

\author{
Impact of resident participation and their role on clinical outcomes of \\ patients undergoing cholecystectomy at a university hospital
}

\author{
Jorge Alberto Martínez-Martínez ${ }^{1} \mathbb{D}$, Hugo Alberto Cómbita-Rojas ${ }^{2} \mathbb{D}$, \\ Pilar Carola Pinillos-Navarro ${ }^{3} \mathbb{D}$, Daniela Casallas-Cristancho ${ }^{4} \mathbb{D}$, Lorena Alejandra Paez-Sosa ${ }^{5} \mathbb{D}$, \\ Sandra Milena Ruiz-Gómez ${ }^{6} \mathbb{D}$
}

1 Médico, residente de Cirugía general, Universidad Nacional de Colombia, Bogotá, D.C., Colombia.

2 Médico, especialista en Cirugía general, profesor asistente, Departamento de cirugía general, Universidad Nacional de Colombia, Bogotá, D.C., Colombia.

3 Médico, especialista en Cirugía general, Universidad Nacional de Colombia, Bogotá, D.C., Colombia.

4 Médico, Universidad Nacional de Colombia, Bogotá, D.C., Colombia.

5 Estudiante de medicina, Universidad Nacional de Colombia, Bogotá, D.C., Colombia.

6 Médico, especialista en Epidemiología, Pontificia Universidad Javeriana, Bogotá, D.C., Colombia.

\section{Resumen}

Introducción. La colecistectomía es uno de los procedimientos quirúrgicos más realizados a nivel mundial, por lo que su aprendizaje es cada vez más necesario para los médicos residentes en entrenamiento, pero sin comprometer la seguridad de los pacientes. El objetivo de este estudio fue determinar el impacto de la participación de los médicos residentes en los principales desenlaces clínicos de la colecistectomía.

Métodos. Se realizó un estudio prospectivo de cohortes, donde se incluyeron los pacientes llevados a colecistectomía laparoscópica, desde junio de 2019 hasta julio de 2020. Se llevó a cabo el análisis estadístico para describir medidas de frecuencia, tendencia central, dispersión y análisis bivariados para los desenlaces de interés.

Resultados. Se incluyeron 482 pacientes a quienes se les practicó colecistectomía, 475 de ellas por vía laparoscópica. El 62,5 \% fueron mujeres y el 76,2 \% se realizaron de carácter urgente. En el $96 \%$ de los procedimientos se contó con la participación de un residente. En el análisis bivariado no se encontró una diferencia estadísticamente significativa entre la participación del residente y un impacto negativo en los desenlaces clínicos de las variables relevantes.

Fecha de recibido: 16/09/2020 - Fecha de aceptación: 15/11/2020 - Fecha de publicación en línea: 06/04/2021

Correspondencia: Jorge Alberto Martínez, Carrera 68 D \# 24B - 48, Bogotá, D.C., Colombia. Teléfono: 3174027274

Dirección electrónica: joramartinezmar@unal.edu.co

Citar como: Martínez-Martínez JA, Cómbita-Rojas HA, Pinillos-Navarro PC, Casallas-Cristancho D, Paez-Sosa LA, Ruiz-Gómez SM. Impacto de la participación del residente y su rol en desenlaces clínicos de los pacientes llevados a colecistectomía en un hospital universitario. Rev Colomb Cir. 2021;36:462-70. https://doi.org/10.30944/20117582.859

Este es un artículo de acceso abierto bajo una Licencia Creative Commons - BY-NC-ND https://creativecommons.org/licenses/by-nc$\mathrm{nd} / 4.0 /$ deed.es 
Discusión. No hay evidencia de que la participación de médicos residentes en la colecistectomía laparoscópica se asocie con desenlaces adversos en los pacientes, lo que sugiere estar en relación con una introducción temprana y responsable a este procedimiento por parte de los docentes, permitiendo que la colecistectomía sea un procedimiento seguro.

Palabras clave: colecistectomía laparoscópica; colelitiasis; cirugía general; programas de postgrado; educación médica; impacto; complicaciones.

\begin{abstract}
Introduction. Cholecystectomy is one of the most performed surgical procedures worldwide, so its learning is increasingly necessary for resident physicians in training, but without compromising the safety of patients. The objective of this study was to determine the impact of the participation of resident physicians on the main clinical outcomes of cholecystectomy.
\end{abstract}

Methods. A prospective cohort study was performed, which included patients undergoing laparoscopic cholecystectomy from June 2019 to July 2020. Statistical analysis was carried out to describe measures of frequency, central tendency, dispersion, and bivariate analysis for outcomes of interest.

Results. 482 patients who underwent cholecystectomy were included, 475 of them laparoscopically; $62.5 \%$ were women and $76.2 \%$ were performed urgently, and $96 \%$ of the procedures involved the participation of a resident. In the bivariate analysis, no statistically significant difference was found between resident participation and a negative impact on the clinical outcomes of the relevant variables.

Discussion. There is no evidence that the participation of resident physicians in laparoscopic cholecystectomy is associated with adverse outcomes in patients, which suggests being related to an early and responsible introduction to this procedure by teachers, allowing cholecystectomy to be a safe procedure.

Keywords: laparoscopic cholecystectomy; cholelithiasis; general surgery; health postgraduate programs; medical education; impact; complications.

\section{Introducción}

La enfermedad litiásica biliar es una de las enfermedades con mayor prevalencia a nivel mundial y la colecistectomía una de las cirugías más frecuentemente realizadas por los cirujanos generales, en Colombia y en el mundo. En la población estadounidense se estima que la prevalencia de esta patología oscila entre el 10 y el $15 \%$ y se realizan más de 700.000 colecistectomías al año. En Colombia no hay datos estadísticos específicos, pero se estima para esta patología una prevalencia de 8,6 \% en centros de referencia en Bogotá ${ }^{1}$.

A raíz de la globalización de los procedimientos laparoscópicos, su uso ha ido en aumento debido a sus ventajas, como menor dolor postoperatorio y menor estancia hospitalaria, lo que ha conllevado a nivel mundial en los últimos años, a una reducción de la cirugía por vía abierta en patologías como la colecistitis. Como consecuencia, la formación de los futuros cirujanos se ha modificado para alcanzar estos objetivos, con un adecuado aprendizaje de las nuevas técnicas desarrolladas, permitiendo que aquellos procedimientos que antes eran realizados exclusivamente por el cirujano adscrito ahora puedan ser realizados por los médicos residentes, respetando los diferentes niveles de competencias, habilidades y complejidad técnica.

Estas modificaciones responden también a la importancia de la participación, cada vez más activa, de los médicos residentes en los procedimientos y el seguimiento de los pacientes, para el desarrollo de sus habilidades, por eso, es importante tener en cuenta el impacto que esto pueda 
tener dentro del sistema de salud, y en el paciente directamente ${ }^{2}$.

Para ello se ha sugerido la importancia de la evaluación y autoevaluación continua de las habilidades de los residentes, ya que se ha demostrado que existe una brecha entre la percepción de los cirujanos adjuntos y los médicos residentes sobre las habilidades laparoscópicas de estos últimos, donde se tiende a subestimar su propio desempeño y habilidades en cirugía, por lo que se ha recomendado un entrenamiento de simulación más estricto y una progresión más temprana a salas de cirugía, para darles la oportunidad de identificar sus debilidades y aumentar su nivel de confianza ${ }^{3-4}$.

Es importante recalcar que la mayoría de estos estudios han sido realizados en países desarrollados, donde la estructura de los programas quirúrgicos tiende a variar, incluso desde la formación de pregrado, por lo que es necesario hacer un análisis objetivo acerca del papel que cumple el residente dentro de las colecistectomías laparoscópicas en nuestro país, para estudiar los factores y desenlaces relacionados con su participación en los diferentes momentos en la estancia hospitalaria de nuestros pacientes, logrando así una adecuada retroalimentación, tanto al personal en formación como a los docentes, para ofrecer una mejor calidad en la atención de los pacientes y en la formación académica de los estudiantes del programa.

El objetivo de este estudio fue determinar el impacto que tiene la participación de los médicos residentes en el acto quirúrgico (colecistectomía) sobre los principales desenlaces, como tiempo quirúrgico, conversión a cirugía abierta, reintervenciones y complicaciones, que determinan a su vez el curso clínico de los pacientes en nuestra institución, con el fin de optimizar la participación e inclusión temprana de los médicos residentes a la cirugía laparoscópica.

\section{Métodos}

Se realizó un estudio prospectivo de cohorte donde se incluyeron los pacientes mayores de 18 años llevados a cirugía por patología de la vesícula biliar, desde junio 2019 hasta julio de 2020, en el Hospital Universitario Nacional de Colombia, centro de alto nivel en la ciudad de Bogotá. Se excluyeron del estudio aquellos pacientes que fueron llevados a cirugía electiva por vía abierta y los pacientes en que no se encontró información completa de su estancia hospitalaria.

Los datos fueron almacenados en Redcap, para garantizar la seguridad de la información de los pacientes; el formato de registro por paciente consta de cinco formularios, con los datos de ingreso, cirugía, egreso, control posquirúrgico y patología. Para el análisis estadístico se utilizó el programa Stata MP, donde los datos fueron organizados en tablas de distribución de frecuencia y se calcularon medidas de tendencia central y de dispersión. Se hizo análisis bivariado para determinar la relación entre la participación del residente y los desenlaces clínicos.

\section{Resultados}

Cuatrocientos ochenta y dos pacientes fueron llevados a colecistectomía en el Hospital Universitario Nacional de Colombia, de los cuales 475 fueron sometidos a colecistectomía laparoscópica y los 7 restantes fueron colecistectomías abiertas electivas, por lo cual fueron excluidos del estudio. De nuestra población, 297 (62,5 $\%$ ) fueron mujeres y la edad promedio fue de 53,2 años. El 23,8 \% (n=113) fueron cirugías programadas de forma ambulatoria y el 76,2 $\%(n=362)$ fueron procedimientos catalogados como urgencia (donde se incluyeron aquellos procedimientos diferidos durante la estancia hospitalaria). Doscientos tres pacientes no reportaron comorbilidad alguna, 124 tuvieron un puntaje menor a tres en el índice de comorbilidades de Charlson ${ }^{5}$, mientras que 148 tuvieron un puntaje igual o mayor a tres. La mitad de los pacientes ( $\mathrm{n}=237,49,9 \%)$ cursaron con clínica de colecistitis, y de estos, 126 pacientes (53,2\%) se clasificaron como grado I según las guías de Tokio $^{6}, 100$ pacientes $(42,2 \%)$ como grado II y 11 pacientes $(4,6 \%)$ como grado III. El riesgo quirúrgico de la población, definido por la clasificación ASA (American Society of Anesthesiologists), se presenta en la tabla 1. 
Tabla 1. Características sociodemográficas y clínicas de los pacientes llevados a colecistectomía laparoscópica $(n=475)$

\begin{tabular}{lc}
\hline \multicolumn{1}{c}{ Variable } & Frecuencia (\%) \\
\hline Sexo & $178(37,5 \%)$ \\
Hombre & $297(62,5 \%)$ \\
Mujer & 53,2 \\
Edad (años) & $203(42,7 \%)$ \\
Índice de comorbilidades de Charlson \\
0 & $124(26,1 \%)$ \\
$<3$ & $148(31,2 \%)$ \\
$>3$ & \\
Clasificación ASA & $152(32 \%)$ \\
I & $228(48 \%)$ \\
II & $80(16,8 \%)$ \\
III & $3(0,6 \%)$ \\
IV & $11(2,3 \%)$ \\
Sin información & $237(49,8 \%)$ \\
Colecistitis aguda & $126(53,1 \%)$ \\
Clasificación Tokio I & $100(42,2 \%)$ \\
Clasificación Tokio II & $11(4,7 \%)$ \\
Clasificación Tokio III & \\
Carácter de la cirugía & $113(23,8 \%)$ \\
Programada o electiva & $362(76,2 \%)$ \\
Urgente & $70(60-90)$ \\
Tiempo quirúrgico (minutos) & 80 (60-115) \\
Clasificación de Parkland & \\
I & $96(20,2 \%)$ \\
II & $170(35,7 \%)$ \\
III & $110(23,1 \%)$ \\
IV & $56(11,7 \%)$ \\
V & $41(8,6 \%)$ \\
\hline Sin dato & $2,4 \%)$ \\
\hline
\end{tabular}

*Datos de mediana y rango intercuartílico de acuerdo con la distribución de la población

Durante la cirugía, la mediana del tiempo quirúrgico fue de 70 minutos para las cirugías programadas (RIC: 60-90 minutos) y 80 minutos para las cirugías de urgencia (RIC: 60-115 minutos). La dificultad técnica se describió mediante la clasificación de Parkland ${ }^{7}$, según la cual, 96 pacientes $(20,2 \%)$ se clasificaron como grado I; 170 (35,8 \%), grado II; $110(23,1 \%)$, grado III; 56 (11,8 \%), grado IV, y 41 (8,6 \%) como grado V. Se convirtió a cirugía abierta en 14 (2,9\%) pacientes y la principal causa fue la dificultad para lograr la visión crítica de seguridad ${ }^{8,9}$ debido al proceso inflamatorio agudo $(n=9)$. Las otras causas fueron hemorragia $(n=2)$, dificultad técnica $(n=2)$ y lesión de otras vísceras $(\mathrm{n}=1)$.

Se reportó lesión biliar en 2 pacientes $(0,4 \%)$, cuya cirugía fue catalogada como urgencia, en quienes la clasificación de Parkland fue IV y no representaron una carga de morbilidad alta para el paciente, con índice de complicación de Clavien-Dindo ${ }^{10}$ I en un paciente y IIIA en el otro.

En 456 procedimientos (96\%) se contó con la presencia de al menos un médico residente de cirugía general, cirugía pediátrica o cirugía plástica; en los 19 procedimientos restantes (4\%) se contó con la asistencia de un médico interno. En aquellas cirugías que contaron con la participación de un médico residente, este figuró como cirujano en $233(51,1 \%)$ procedimientos y como primer ayudante en los otros 223 (48,9\%).

Los residentes de primer año participaron en 166 colecistectomías laparoscópicas (34,9\%), los de segundo en $154(32,4 \%)$ y los de tercer año en 136 (28,6 \%) cirugías; el papel desempeñado por cada uno se encuentra descrito en la tabla 2. Es de tener que cuenta que el residente de primer año participó como cirujano predominantemente en colecistectomías con clasificación Parkland 1, mientras que el residente de tercer año y el cirujano, en colecistectomías de mayor dificultad técnica (Parkland 4 y 5).

La mediana del tiempo de estancia hospitalaria para los pacientes sometidos a cirugía de urgencia fue de 5 días (RIC: 4-7 días), mientras que la mayoría de los pacientes que ingresaron para cirugía programada, tuvieron egreso en las siguientes 24 horas. Dentro de las complicaciones se reportó infección del sitio operatorio en 5 casos $(1,1 \%)$,

Tabla 2. Participación y papel del residente quirúrgico la realización de colecistectomías laparoscópicas

\begin{tabular}{lccc}
\hline $\begin{array}{c}\text { Año de } \\
\text { residencia }\end{array}$ & \multicolumn{2}{c}{ Papel desempeñado } & Total (n=456) \\
\hline Primer & $21(4,6 \%)$ & $145(31,8 \%)$ & $166(36,4 \%)$ \\
Segundo & $105(23,0 \%)$ & $49(10,7 \%)$ & $154(33,8 \%)$ \\
Tercer & $107(23,4 \%)$ & $29(6,3 \%)$ & $136(29,8 \%)$ \\
\hline
\end{tabular}


de los cuales 2 fueron incisional superficial, 2 profunda y 1 con compromiso de órgano-espacio; se diagnosticó sepsis secundaria al procedimiento quirúrgico en 4 pacientes $(0,8 \%)$ e íleo postoperatorio en 1 caso $(0,2 \%)$. Adicionalmente, se aplicó la clasificación de Clavien-Dindo, encontrando que $306(64,4 \%)$ pacientes no presentaron complicación alguna; $97(20,4 \%)$ se clasificaron como grado I; 53 (11,1\%), grado II; $6(1,2 \%)$, grado IIIA; $3(0,6 \%)$, grado IIIB; $5(1,1 \%)$, grado IVA; $3(0,6 \%)$, grado IVB, y $2(0,4 \%)$ se clasificaron como grado $\mathrm{V}$.
Catorce pacientes $(2,9 \%)$ requirieron reingreso para manejo hospitalario, de los cuales 6 reingresaron por complicaciones relacionadas con el procedimiento quirúrgico, 4 por alguna complicación médica asociada, 3 por motivos independientes del procedimiento y 1 por dolor en sitio quirúrgico. Se reportaron 3 muertes $(0,6 \%)$ en pacientes que cursaron con colecistitis, 2 de ellos Tokio III y uno Tokio II, a su vez con clasificación ASA III y IV, respectivamente, lo cual concuerda con los desenlaces posibles por las comorbilidades de base.

Tabla 3. Análisis bivariado en pacientes sometidos a colecistectomía laparoscópica según conversión a cirugía abierta y tiempo quirúrgico

\begin{tabular}{|c|c|c|c|c|c|c|}
\hline \multirow[b]{2}{*}{ Parámetro } & \multicolumn{3}{|c|}{ Conversión a cirugía abierta } & \multicolumn{3}{|c|}{ Tiempo quirúrgico } \\
\hline & OR & $\mathrm{IC}_{95 \%}$ & $\mathbf{p}$ & $\mathbf{n}$ & OR & $\mathbf{p}$ \\
\hline Edad & 1,07 & $1,03-1,11$ & $0,182^{* *}$ & 475 & 0,193 & $0,000^{* * * *}$ \\
\hline Sexo: Mujer & 0,79 & $0,28-2,226$ & 0,653 & 178 & 0,567 & $0,014^{*}$ \\
\hline \multicolumn{7}{|l|}{ Índice de Charlson } \\
\hline 0 & - & - & - & 203 & - & - \\
\hline $1-2$ & 4,91 & $0,51-46,59$ & $0,155^{*}$ & 124 & 0,470 & $0,354^{*}$ \\
\hline$>3$ & 13,71 & $1,77-105,98$ & $0,001^{*}$ & 148 & 0,407 & $0,002^{*}$ \\
\hline \multicolumn{7}{|l|}{ ASA } \\
\hline 1 & - & - & - & 152 & - & - \\
\hline II & 4,91 & $0,57-12,38$ & $0,327^{*}$ & 228 & 0,424 & $0,121^{*}$ \\
\hline II -IV & 13,71 & $0,68-19,57$ & $0,189^{*}$ & 83 & 0,341 & $0,000^{*}$ \\
\hline Colecistitis aguda & 1,80 & $0,61-5,31$ & 0,274 & 237 & 0,378 & $0,000^{*}$ \\
\hline \multicolumn{7}{|l|}{ Clasificación de severidad } \\
\hline Tokyo I & - & - & - & 126 & - & - \\
\hline Tokyo II & 3,15 & $0,62-15,89$ & $0,246^{*}$ & 100 & 0,450 & - \\
\hline Tokyo III & 11,45 & $1,78-73,62$ & $0,032^{*}$ & 11 & - & $0,217^{* * *}$ \\
\hline \multicolumn{7}{|l|}{ Clasificación de Parkland } \\
\hline 1 & - & - & - & 96 & - & - \\
\hline $2-3$ & 0,00 & - & $0,576^{*}$ & 280 & 0,325 & $0,000^{*}$ \\
\hline $4-5$ & 0,00 & - & $0,002^{*}$ & 97 & 0,150 & $0,000^{*}$ \\
\hline \multicolumn{7}{|l|}{ Residente en papel de cirujano } \\
\hline Primer año & 0,00 & - & & 21 & - & $0,999^{* * *}$ \\
\hline Segundo año & 0,00 & - & $0,584^{*}$ & 105 & - & $0,362^{* * *}$ \\
\hline Tercer año & - & - & & 107 & - & $0,381^{* * *}$ \\
\hline \multicolumn{7}{|l|}{ Residente en papel de primer ayudante } \\
\hline Primer año & 0,35 & $0,10-1,11$ & & 145 & & $0,088^{* * *}$ \\
\hline Segundo año & 0,14 & $0,01-1,26$ & $0,087^{*}$ & 49 & - & $0,045^{\star * *}$ \\
\hline Tercer año & & & & 29 & 0,224 & $0,001^{*}$ \\
\hline Cirujano sin participación del residente & 0,00 & - & 0,013 & 19 & - & $0,147^{*}$ \\
\hline \multicolumn{7}{|l|}{ Carácter de la cirugía } \\
\hline Urgente & 0,00 & - & $0049 *$ & 362 & 0,395 & $0,000^{*}$ \\
\hline Programada o electiva & 0,00 & - & $0,049^{n}$ & 113 & & \\
\hline
\end{tabular}

* P valor con test de Fisher; ** Test Mann-Whitney; ${ }^{* * *}$ Test de Kolmogorov-Smirnow; ${ }^{* * *}$ Test de Spearman 
En cuanto al análisis bivariado, se encontró que un índice de Charlson > 3 (OR 13,71; IC ${ }_{95 \%}$ : 1,77-105,98), una clasificación de ASA III - IV (OR 3,66; IC $95 \%$ : 0,61-5,31), una clasificación de Tokio III (OR 11,45; IC95\%: 1,78-73,62), el hallazgo intraoperatorio clasificado como Parkland IV-V y una cirugía de urgencia, son factores que se relacionan con un incremento en la probabilidad de conversión a cirugía abierta. Por el contrario, no hubo hallazgos estadísticamente significativos entre este desenlace y el año de residencia o el tipo de participación del residente en el acto quirúrgico (tabla 3).

Por otro lado, en el análisis bivariado para el tiempo operatorio, el sexo masculino $(\mathrm{p}=0,014)$, un índice de Charlson $>3(p=0,002)$, una clasificación de ASA III-IV ( $p=0,000)$, presencia de colecistitis aguda $(\mathrm{p}=0,000)$, una clasificación de Parkland diferente a $1(\mathrm{p}=0,000)$ y una cirugía de urgencia, son factores que se relacionan con un incremento en el tiempo quirúrgico, mientras la participación del médico residente (como ayudante o cirujano) no tuvo relación con la prolongación del tiempo quirúrgico con una diferencia estadísticamente significativa.

\section{Discusión}

A pesar de las múltiples ventajas que aporta la colecistectomía laparoscópica, persiste la preocupación por la temida lesión de la vía biliar, aunque estudios recientes, incluyendo en nuestra institución, han demostrado que ocurre en un porcentaje igual o menor al presentado en cirugía abierta ${ }^{11}$; por ejemplo, Richardson ${ }^{12}$ describió como la lesión de la vía biliar en la colecistectomía laparoscópica ha ido en descenso, siendo de $0,8 \%$ en los años noventa y de $0,4 \%$ en el nuevo milenio, un dato que ha sido comprobado por otros estudios más recientes, donde la tasa de lesión de vía biliar en la mayoría de los estudios es de 0,38-0,4 \% ${ }^{13-15}$. Esto puede relacionarse con el aumento de las competencias de los cirujanos y la introducción de conceptos como la visión crítica de seguridad, en 1995, por el doctor Strasberg ${ }^{8}$.

En estudios realizados en centros de cuarto nivel de atención en Colombia, se han estudiado las complicaciones encontrando tasas de lesión de vía biliar entre $0,4 \%$ y $1,1 \%{ }^{16,17}$, sin embargo, en análisis previos de nuestra institución ${ }^{8}$, se encontró una tasa menor, siendo esta del 0,25 \%, así como una menor tasa de conversión a cirugía abierta (3,3 \%) en 24 meses de seguimiento, un valor que contrasta con otros estudios realizados en el país, donde el porcentaje de conversión a cirugía abierta reportado oscila entre el 4,3 \% y el $20 \%{ }^{18}$, lo cual indica que este es un procedimiento seguro, y por lo tanto, de elección para la patología biliar en nuestra institución ${ }^{8}$.

En la revisión de la literatura, no encontramos estudios en nuestro país que evalúen el impacto de la participación de los médicos residente en los desenlaces clínicos de los pacientes, por lo que consideramos el presente estudio de gran importancia para iniciar una evaluación objetiva del desempeño de estos médicos en entrenamiento y el impacto del mismo, para poder generar estrategias acordes a los resultados.

En un análisis realizado en diferentes centros estadounidenses, que incluyó 71.890 cirugías básicas, de las cuales 37.636 correspondían a colecistectomías laparoscópicas, se encontró que la participación del médico residente no resultó en un aumento significativo de las probabilidades de mortalidad, morbilidad o reintervención, así como tampoco hubo cambios en el tiempo de estancia hospitalaria ni en la tasa de readmisión ${ }^{2}$, aunque sí podría aumentar el tiempo quirúrgico ${ }^{19}$. Algunos estudios, como el de Jolley y colaboradores, sugieren que el tiempo operatorio durante la participación del residente podría aumentarse del $19 \%$ al $47 \%$, y podría relacionarse con mayores tasas de morbilidad postoperatoria, como de infección del sitio quirúrgico ${ }^{20-21}$.

Por otro lado, Tseng ${ }^{22}$ reportó que la participación de los residentes de cirugía se asoció con una probabilidad $14 \%$ mayor de morbilidad, pero una probabilidad $58 \%$ menor de mortalidad, lo que se complementa con lo descrito por Jolley, sobre que la presencia de médicos residentes atentos beneficia a los pacientes quirúrgicos en lo que respecta a los cuidados postoperatorios y la reducción general de las tasas de mortalidad ${ }^{2}$. 
Encontramos datos interesantes que van en concordancia con la mayoría de la literatura reportada. Nuestro análisis bivariado para la conversión a cirugía abierta mostró relación de características propias del cuadro clínico y del paciente, pero no de la participación ni el rol de cirujano por parte del residente. En cuanto a las complicaciones generales, se observó que el papel del residente como cirujano no aumenta la incidencia de las mismas, con un valor de $\mathrm{p}>0,5$ para la correlación del rol con complicaciones del procedimiento. Por el contrario a lo encontrado en la literatura mundial, el análisis bivariado de esta cohorte demostró que el tiempo operatorio no se ve afectado por la participación del residente, tanto como cirujano como ayudante.

Una de las fortalezas de los análisis previos es contar con un grupo de pacientes intervenidos quirúrgicamente sin la presencia del residente, con el instructor como cirujano, ya que puede hacer como grupo de control en cuanto a conversión, tiempo quirúrgico y complicaciones, las cuales no aumentaron al permitir la participación del residente. La ocurrencia de desenlaces adversos como mortalidad y lesión de la vía biliar fue muy baja, por lo que no se pueden realizar inferencias con adecuado valor estadístico, aunque se debe resaltar que se encuentran dentro de los porcentajes que mantienen los estándares internacionales para estos desenlaces.

Es necesario relacionar los hallazgos descritos previamente con la distribución de la dificultad técnica de la cirugía que predominó en cada grupo; siendo clasificados como Parkland 1 y 2 los procedimientos realizados por los residentes de los primeros años, mientras que los procedimientos realizados por los residente de tercer año y los cirujanos generales fueron clasificados como Parkland 4 y 5 . Esto puede explicar el hecho que no aumentó el tiempo operatorio, la conversión a cirugía abierta y las complicaciones, ya que el rol del médico residente va de acuerdo con las competencias y habilidades propias para cada nivel. En las cirugías practicadas por los instructores, en su rol de cirujano, no se presentó conversión a cirugía abierta y mantuvieron una mediana de tiempo operatorio similar, ya que intervinieron en los procedimientos de mayor dificultad técnica y en pacientes de más alto riesgo.

\section{Limitaciones}

El presente estudio se trata de un análisis prospectivo de un solo centro de atención, lo cual puede disminuir la validez externa de los resultados, haciendo que se requieran estudios individuales en cada institución. A su vez, presenta limitación por el número de pacientes y por la naturaleza de recolección prospectiva reciente, pero se realizó un control adecuado de los sesgos de información, ya que la base fue diseñada de forma dirigida al estudio, presentando una calidad de registro adecuada en las variables.

\section{Conclusión}

En nuestra institución, un hospital universitario de alta complejidad, la participación de los residentes de primer, segundo y tercer año, tanto de los cirujanos como primer ayudante, en las cirugías de colecistectomía laparoscópica, no aumentó el tiempo quirúrgico, la conversión a cirugía abierta o las complicaciones generales. Se puede establecer que estos resultados tienen relación con la introducción temprana de los médicos residentes a la cirugía laparoscopia, según habilidades y competencias propias de cada nivel de residencia, como se demostró en esta cohorte. Se considera que la colecistectomía laparoscópica con intervención del médico residente es un procedimiento seguro en nuestro centro. Se requieren estudios adicionales para generar estrategias encaminadas a mejorar la participación de los residentes y con ello, el desenlace de los pacientes.

\section{Cumplimiento de normas éticas}

Consentimiento informado: De acuerdo con lo establecido en la resolución 08430 de 1993 del Ministerio de Salud en el cual se establecen las normas científicas, técnicas y administrativas para la investigación en salud, el presente estudio es una investigación sin riesgo en el cual no se realizó ninguna intervención en los individuos que participaron en el estudio. Se realizó una revisión de las historias clínicas y de base de datos de la información 
registrada siempre preservando la seguridad de la información de los pacientes.

Conflicto de interés: Ninguno declarado por los autores.

Fuentes de financiamiento: El estudio financiado con recursos de los autores.

\section{Contribución de los autores:}

- Concepción y diseño del estudio: Jorge Alberto Martínez-Martínez, Hugo Alberto Cómbita-Rojas, Pilar Carola Pinillos, Daniela Casallas-Cristancho.

- Adquisición de datos: Lorena Alejandra Páez, Jorge Alberto Martínez-Martínez, Daniela CasallasCristancho, grupo de investigación en cirugía general de la facultad de medicina de la Universidad Nacional de Colombia.

- Análisis e interpretación de datos: Jorge Alberto Martínez-Martínez, Daniela Casallas-Cristancho, Sandra Milena Ruiz-Gómez.

- Redacción del manuscrito: Jorge Alberto MartínezMartínez, Daniela Casallas-Cristancho, Lorena Alejandra Páez.

- Revisión crítica: Hugo Alberto Cómbita-Rojas, Pilar Carola Pinillos.

\section{Referencias}

1. Vázquez RS. Colecistopatías. En: Vargas A. Gastroenterología. Segunda edición. México: Mc Graw-Hill Interamericana; 1998. p. 2.

2. Jolley J, Lomelin D, Simorov A, Tadaki C, Oleynikov D. Resident involvement in laparoscopic procedures does not worsen clinical outcomes but may increase operative times and length of hospital stay. Surg Endosc. 2015;30:3783-91. https://doi.org/10.1007/s00464-015-4674-z

3. Quick JA, Kudav V, Doty J, Crane M, Bukoski AD, Bennett BJ, Barnes SL. Surgical resident technical skill self-evaluation: increased precision with training progression. J Surg Res. 2017;218:144-9. https://doi.org/10.1016/j.jss.2017.05.070

4. Alameddine MB, Claflin J, Scally CP, Noble DM, Reames BN, Englesbe MJ, Wong SL. Resident surgeons underrate their laparoscopic skills and comfort level when compared with the rating by attending surgeons. Journal Of Surgical Education. 2015;72:1240-6. https://doi.org/10.1016/j.jsurg.2015.07.002

5. Charlson ME, Pompei P, Ales KL, MacKenzie CR. A new method of classifying prognostic comorbidity in longitudinal studies: development and validation. J Chronic Dis. 1987;40:373-83. https://doi.org/10.1016/0021-9681(87)90171-8
6. Yokoe M, Hata J, Takada T, Strasberg SM, Asbun HJ, Wakabayashi G, et al. Tokyo Guidelines 2018: diagnostic criteria and severity grading of acute cholecystitis (with videos). J Hepatobiliary Pancreat Sci. 2018;25:41-54. https://doi.org/10.1002/jhbp.515

7. Madni TD, Leshikar DE, Minshall CT, Nakonezny PA, Cornelius CC, Imran JB, et al. The Parkland grading scale for cholecystitis. Am J Surg. 2018;215:625-30. https://doi.org/10.1016/j.amjsurg.2017.05.017

8. Strasberg SM, Hertl M, Soper NJ. An analysis of the problem of biliary injury during laparoscopic cholecystectomy. J Am Coll Surg. 1995;180:101-125.

9. Strasberg SM, Brunt LM. Rationale and use of the critical view of safety in laparoscopic cholecystectomy. J Am Col Surg. 2010;211:132-8.

https://doi.org/10.1016/j.jamcollsurg.2010.02.053

10. Clavien PA, Barkun J, de Oliveira ML, Vauthey JN, Dindo D, Schulick RD, et al. The Clavien-Dindo classification of surgical complications: five-year experience. Ann Surg. 2009;250:187-96.

https://doi.org/10.1097/SLA.0b013e3181b13ca2

11. Pinillos-Navarro P, Junca-Burgos E, Buitrago-Gutiérrez G. Factores pronósticos asociados con desenlaces clínicos de los pacientes llevados a colecistectomía en El Hospital Universitario Nacional De Colombia: Estudio de cohortes. Tesis de grado para Especialidad en Cirugía General. Universidad Nacional de Colombia. 2019

12. Richardson MC, Bell G, Fullarton GM. Incidence and nature of bile duct injuries following laparoscopic cholecystectomy: an audit of 5913 cases. West of Scotland laparoscopic cholecystectomy audit group. Br J Surg. 1996;83:1356-60. https://doi.org/10.1002/bjs.1800831009

13. Pucher PH, Brunt LM, Davies N, Linsk A, Munshi A, Rodriguez HA, et al. Outcome trends and safety measures after 30 years of laparoscopic cholecystectomy: a systematic review and pooled data analysis. Surg Endosc. 2018;32:2175-83. https://doi.org/10.1007/s00464-017-5974-2

14. Kohn JF, Trenk A, Kuchta K, Lapin B, Denham W, Linn JG, et al. Characterization of common bile duct injury after laparoscopic cholecystectomy in a high-volume hospital system. Surg Endosc. 2018;32:1184-91. https://doi.org/10.1007/s00464-017-5790-8

15. Diamantis T, Tsigris C, Kiriakopoulos A, Papalambros E, Bramis J, Michail P, et al. Bile duct injuries associated with laparoscopic and open cholecystectomy: an 11-year experience in one Institute. Surg Today. 2005;35:841-5. https://doi.org/10.1007/s00595-005-3038-z

16. Aldana GE, Martínez LE, Hosman MA, Ardila DA, Mariño IF, Sagra MR, Montoya LM. Factores predictores perioperatorios de complicaciones de la colecistectomía por 
laparoscopia. Rev Colomb Cir. 2019;33:162-72. https://doi.org/10.30944/20117582.58

17. Domínguez LC, Herrera WE, Rivera AM, Bermúdez CE. Colecistectomía de urgencia por laparoscopia por colecistitis aguda en adultos mayores. Rev Colomb Cir. 2011;26:93-100.

18. Chinchilla PA, Baquero DR, Ruiz JE. Factores de riesgo preoperatorios asociados a conversión a técnica abierta en colecistectomía laparoscópica de urgencia. Rev Colomb Cir. 2018;33:145-53.

https://doi.org/10.30944/20117582.56

19. Uecker J, Luftman K, Ali S, Brown C. Comparable operative times with and without surgery resident participation. J Surg Educ. 2013;70:696-9. https://doi.org/10.1016/j.jsurg.2013.06.011
20. Davis SS, Husain FA, Lin E, Nandipati KC, Perez S, Sweeney JF. Resident participation in index laparoscopic general surgical cases: impact of the learning environment on surgical outcomes. J Am Coll Surg. 2013;216:96-104.

https://doi.org/10.1016/j.jamcollsurg.2012.08.014

21. Papandria D, Rhee D, Ortega G, Zhang Y, Gorgy A, Makary MA, Abdullah F. Assessing trainee impact on operative time for common general surgical procedures in ACS-NSQIP. J Surg Educ. 2012;69:149-155. https://doi.org/10.1016/j.jsurg.2011.08.003

22. Tseng WH, Jin L, Canter RJ, Martinez SR, Khatri VP, Gauvin J, et al. Surgical resident involvement is safe for common elective general surgery procedures. J Am Coll Surg. 2011;213:19-26.

https://doi.org/10.1016/j.jamcollsurg. 2011.03.014 